[REVIEW ARTICLE]

\title{
Preparing to meet the oral health needs of the elderly in Qatar - A model for Domiciliary Oral Health Care Services
}

\author{
Najat Abdrabbo AlYafei ${ }^{1,}{ }^{*}$ and Bushra Naaz Fathima Jaleel 2 \\ ${ }^{1}$ Head of Oral Public Health, Dentistry Department, Primary Health Care Corporation, Doha, Qatar. \\ 2 Oral Public Health Coordinator, Dentistry Department, Primary Health Care Corporation, Doha, Qatar.
}

Publication history: Received on 01 December 2020; revised on 12 December 2020; accepted on 14 December 2020

Article DOI: https://doi.org/10.30574/wjarr.2020.8.3.0459

\begin{abstract}
Aging is a natural and irreversible process of life. Oral health of elderly people is an important public health issue and good oral health is an essential part of their health care. Currently, the challenge lies in aligning the existing health system with the needs and preferences of the elderly people.

The aim of this article is to propose a model for Domiciliary Oral Health Care Services for elderly in Qatar, wherein a definite pathway for oral care is identified and the Domiciliary Oral Health Care Services program is standardized, from the initial phase of oral assessment, through the oral health promotion and preventative phase until the dental treatment phase. This model will help to deliver oral health care to elderly who may be unable to access or face difficulties to access the dental services in conventional dental clinical settings due to disability, infirmity or old age. It will ensure oral comfort, pain relief, essential oral care and enhancement of oral hygiene for the elderly. Working in collaboration with organizations offering Home Healthcare Services, Domiciliary Oral Health Care Services will help in realization of the shared goal of achieving 'Healthy Ageing', holistic health and welfare for the elderly in Qatar. By favoring the policy to initiate the DOHCS as "Essential health services that benefits all elderly people living in Qatar", the State of Qatar would probably be the pioneer in Middle East region to initiate such a program at National level for improving the oral health of the elderly.
\end{abstract}

Keywords: Domiciliary Oral Health Care; Elderly; Oral Health; Homecare; Qatar

\section{Introduction}

Qatar is a fascinating mosaic of the ultra-modern and vibrant traditional cultures. Qatari traditions are strongly rooted and influenced by the Bedouin culture where family bonds, religion and customs exemplify as integral components and are highly valued. Both, Islamic teaching and Qatari culture emphasize the respect for parents and elders, while recognizing the quintessential rights of the elders upon the younger generations as they approach old age and senility. Qatar has demonstrated its commitment to enhance the health care, social services and empowerment for older adults by prioritizing 'Healthy Ageing' as a goal for the National Health Strategy 2018 - 2022 [1]. Several organizations are channeling their resources to ensure that the elderly are catered to, feel at ease and reassured to lead a contented life during their golden years.

$1.5 \%$ of Qatar's population is aged 65 years and above [2], with an average life expectancy at birth of 80.7 years [3]. Advances in medicine have increased the likelihood that people today live longer with comorbidities than their predecessors [4]. The old age dependency ratio, which is the ratio of individuals older than 64 years dependents on the working-age population aged 15 to 64 , is computed to be $1.9 \%$ in 2019 and projected to reach $6 \%$ by 2030 [2] and

\footnotetext{
* Corresponding author: Najat Abdrabbo AlYafei

Head of Oral Public Health, Dentistry Department, Primary Health Care Corporation, Doha, Qatar.
} 
16.6\% by 2050 in Qatar [5]. This demographic trend also presents challenges to the health care system as it must be equipped to manage their senility and chronic disorders, while contemporaneously being able to foresee, prevent and treat potential acute diseases. A crucial point of interest for the elderly is the provision of state-of-the-art prevention and health promotion to ensure improved quality of life through delivery of targeted, safe, effective, acceptable and empathetic home care.

\section{Home Healthcare Services in Qatar}

Aging is a natural and irreversible process of life, often accompanied by an assortment of diverse and complex medical, physical, and psychological conditions that may limit the person's ability to carry out daily activities and increase their dependency on family members and care givers.

Several Governmental and Non-Governmental Home Healthcare Services [HHS] organizations in Qatar continuously strive to maintain and improve the health as well as quality of life of care receivers with diverse needs. They offer an array of services ranging from nursing, physiotherapy, post-operative care, elderly care, laboratory services, pregnancy, maternity and newborn baby care to health education for caregivers and family.

Since the time of the launch of Home Healthcare Services in Hamad Medical Corporation [HMC] in 2003 and Primary Heath Care Corporation [PHCC] in 2014, these two principal public health care providers deliver optimum home healthcare and continue to expand in their coverage and capacity. Currently more than 1,850 elderly patients receive home healthcare every month through HMC and PHCC alone [6]. HHS for the elderly mainly focus on providing convenient health care services for the elderly in their homes where they are more relaxed, comfortable and feel secure in contrast to the stressful and threatening experience they may perceive in a clinic or hospital setting.

Provision of oral health care services will further propel the versatile HHS to achieve holistic health for the elderly in Qatar. Working in collaboration with all the organizations offering home healthcare services will help in realization of the shared goal of achieving overall health and welfare for the elderly in Qatar. However, there is hardly any evidence of organized oral health care services being delivered as part of HHS for this segment of the population.

The aim of this article is to propose a model for Domiciliary Oral Health Care Services for elderly, abbreviated by the acronym DOHCS, wherein the definite pathway for oral care is identified and the program is standardized, from the initial phase of oral assessment, through the oral health promotion and preventative phase until dental treatment phase, so as to achieve improvement in the oral health of this priority group. Once this initiative gains traction, it can be established as a formalized National Health Program of DOHCS for the elderly in Qatar.

\section{What are DOHCS?}

Domiciliary Care has been defined as, "A service that reaches out to care for those who cannot reach the service themselves" [7]. Accordingly, domiciliary dental care is defined as, "the provision of dental care in an environment where a person is a resident either permanently or temporarily, as opposed to dental care delivered in a fixed dental clinic or a mobile dental unit"[8].

\section{Need for DOHCS}

The elderly generally experiences poor oral health due to a high prevalence of dental caries, periodontal disease, root caries, tooth loss, in addition xerostomia, oral candidiasis, mucositis, cracked lips, fissured tongue, oral precancer and precancer [11]. Old age is also characterized by the emergence of several complex health states and coexisting chronic diseases like osteoarthritis, diabetes, hypertension, chronic obstructive pulmonary disease, depression, and dementia. Moreover, long-term conditions like arthritis, Parkinson's disease, dementia etc. can contribute to inadequate oral hygiene maintenance and/or act as barriers to access the dental clinic for treatment. Many prescriptions and/or over the counter medications taken by older people produce side effects like xerostomia, which increases the risk of root caries [12].

Furthermore, the interrelationship between oral health and general health is more particularly pronounced among older people. Severe periodontal disease is often associated with diabetes mellitus, ischemic heart disease and chronic respiratory disease, while poor oral hygiene predisposes to bacterial endocarditis [13] and aspirational pneumonia [14]. Tooth loss has also been linked with increased risk of ischemic stroke and poor mental health [11]. Hence oral care 
for dependent elderly people should be prioritized as a collaborative action with other health professionals, considering the fact that many conditions in the oral cavity have direct interference on their systemic health [15].

Health needs of the elderly are unique and require specialized care to address the many normal physiological, social and emotional changes of aging, as well as the multitude of acute and chronic manifestations of illness encountered in this age group [16]. Oral health of elderly people is an important public health issue and good oral health is an essential part of their health care. Currently, the challenge lies in aligning the existing health system with the needs and preferences of the elderly people. Moreover, as stated by Simon, "The separation of dental and medical care is a medical ethics issue because it negatively impacts vulnerable populations who lack access to dental care" [17]. Given the well documented association, dental care for the dependent elderly should be prioritized as an interdisciplinary health action [15].

Several successful oral health prevention programs and interventional strategies lend support for improving the oral health and oral health-related quality of life in elderly people [18-21]. Well established DOHCS which have proved to be effective for the Community based Oral Health Promotion are flourishing in countries like Australia [10], China [22], Europe [23] and the United Kingdom [8,24].

\section{Advocacy for National DOHCS program}

By favoring the policy to initiate the DOHCS as "Essential health services that benefits all elderly people living in Qatar", the State of Qatar would probably be the pioneer in Middle East region to initiate such a program for improving the oral health of the elderly by enabling easy access to oral health care services from their home. DOHCS Coordinator can perform operational coordination of care for the program at National level under the Ministry of Public Health, once the DOHCS program is established as National Health Program.

\section{Objectives of DOHCS}

- To raise awareness about oral health assessment finding to the elderly, their family, or caregivers and recommend Domiciliary Oral Health Care services.

- To deliver oral health care to elderly who may be unable to access or face difficulties to access the dental services in conventional dental clinical settings due to disability, infirmity or old age.

- $\quad$ To ensure the maintenance of good oral hygiene among elderly seeking DOHCS.

- To deliver high quality care in a person-centered way that respects the dignity of the individual receiving it.

- To establish interprofessional collaboration of PHCC- DOHCS team with other Home healthcare providers.

- To standardize the DOHCS guidelines and protocol and facilitate development of National DOHCS Policy and Program to expand these integrated services with an effective framework for benefit of its recipients, without duplication of efforts and resources.

\section{Domiciliary Oral Health Care Services model}

DOHCS will be provided to elderly and older adults taking into consideration their age, nature of the dental problem and facilities available, to ensure overall welfare by improving their oral comfort, relieving oral pain and improving their oral hygiene. It is proposed that this integrated DOHCS model which includes oral health assessments, delivery of preventive measures and decisions for dental treatments will significantly benefits the older population. Through DOHCS access to dental care is ensured for elderly, who may not able to visit the dental office. The current DOHCS model is designed to be implemented in 3 phases to provide essential oral care to the elderly in Qatar [Figure 1].

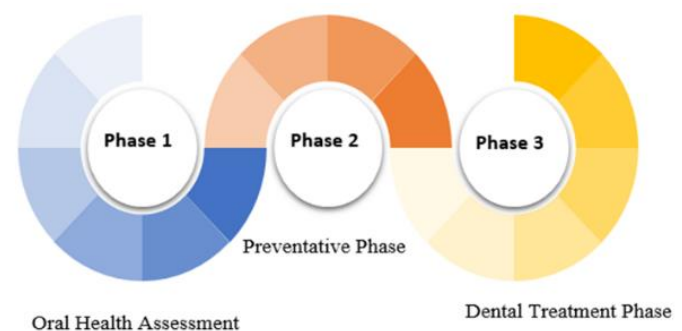


Figure 1 Phases of Domiciliary Oral Health Care Services

\subsection{Phase 1: Oral Health Assessment:}

DOHCS are envisaged to be embedded and integrated within Primary Health Care [PHC] by operating a dedicated DOHCS telephone outcall program and helpline. DOHCS Coordinators would be the focal persons to deliver information about these support services by linking with the geriatric population registered for Home Healthcare Services at Primary Health Care Centers. Despite the high prevalence of oral problems in old age, dental attendance by the frail older population is low. The role of non-dental health care providers, like DOHCS Coordinators in assessing and promoting oral health among elderly becomes imperative [25].

The telephone out call program will connect with the elderly people registered for Home Healthcare Services at Hamad Medical Corporation [HMC] and other private Homecare organizations. Additionally, any elderly individual, their family members, caregivers or organizations who intend to benefit from DOHCS, can initiate contact with DOHCS Coordinators through the toll-free helpline.

Oral Health Assessment Tool [OHAT] employed for initial need assessment is simple and does not require clinical examination but enquires about dentition status, experience of pain and Oral Health Related Quality of Life [OHRQoL] of the elderly. The potential eligibility of the elderly for DOHCS will be evaluated by DOHCS Coordinator by appraising 6 questions, adapted from Better Oral Health in Home Care [BOHHC] model [26].

- Do you have any of your own natural teeth?

- Have you had pain in your mouth while chewing?

- Have you lost any fillings, or do you need a dental visit for any other reason?

- Have you avoided laughing or smiling because of problems with your teeth, mouth or dentures?

- Have you had to interrupt meals because of problems with your teeth, mouth or dentures?

- Have you had difficulty relaxing because of problems with your teeth mouth or dentures?

An affirmation to any one of these questions indicates need for DOHCS. The same will be endorsed by the DOHCS Coordinator during the phone call and discussed with the elderly and his/her family/caregivers based on findings of the assessment and their consent for DOHCS will be enquired. The DOHCS Coordinator would then appraise the DOHCS team comprising of Dental Hygienists and Dentists and initiate the next phase of the services.

\subsection{Phase 2: Preventative Phase}

Prevention and Oral Health Promotion are given utmost priority in DOHCS to reduce the likelihood of development of further oral problems. DOHCS Dental Hygienists will visit the elderly at their home. Effective use of dental auxiliaries has proven to reduce plaque scores and shown improvement of referral pathways for complex dental treatment needs [27]. On obtaining the signed informed consent they will record the medical and dental history, conduct a comprehensive oral examination and assessment of treatment needs, besides essential preventative oral care. This includes oral hygiene instructions [brushing instructions, modified oral hygiene aids, electric tooth brushes, choice of tooth paste, use of mouthwash], instructions to maintain denture hygiene in edentulous patients, dietary and nutritional advise, advise to quit smoking, shisha and other deleterious habits as well as behavior management to improve their compliance with DOHCS, while constantly motivating and guiding them to maintain optimal oral hygiene.

Of prime importance in this phase is the Oral Health Education and the personalized oral care plan tailored to the individual's needs, which will be delivered to the elderly and/or in case of extreme frailty and dependency to their family members or caregivers. This will empower them to maintain their oral health.

The oral findings of concern will be accurately documented and photographed for further reference. At this stage a tele dentistry consultation with the DOHCS Dentist may also be arranged and the provisional treatment plan will be discussed with elderly and/or a caregiver. Some elderly people may also require a physician consultation for fitness to undergo dental treatment in case of systemic diseases and to discuss possible pharmacological interactions. Concomitantly, the ambulatory status of the patient is assessed by the Dental Hygienist and conveyed to the DOHCS coordinator. Further dental treatment is planned accordingly taking into consideration their age, physical condition and the nature of their dental problem. 


\subsection{Phase 3: Dental Treatment Phase}

DOHCS aims to provide essential dental care, eliminate any focus of infection or pain and improve the oral health and comfort. For non-ambulatory patients, the assigned DOHCS Dentist and Dental Assistant will make a home visit. They will deliver the dental treatment, based on the need of the patient, using Mobile Dental Unit following adequate infection control protocol. For ambulatory patients all the appropriate dental treatment will be delivered at the Dental clinics in the Primary Health Care Centers by allocating prioritized appointment slots, and/or pertinent referral to HMC for the specialized/ secondary dental treatment.

The DOHCS Dental Team will be trained to be proficient in gerontology, chronic diseases and management of their oral manifestations and medical emergencies. They will be competent in delivery of oral care in a non-clinical setting. They will also be trained to communicate effectively and develop a rapport with older people, their families and caregivers by empathetic attitude and employing reflective listening.

Essential equipment for DOHCS at patient's home will comprise of portable dental chair, portable dental light, treatment unit, Personal Protective Equipment [surgical masks, gloves, gown and head covers], Dental kits; [1] General examination kit: Mouth mirrors, explorers, periodontal probes, cotton, gauze, tweezer [2]. Medical kit: Topical anesthetic spray, local anesthesia injections, analgesic and anti-inflammatory drugs, emergency medicines [3]. Periodontal kit: ultrasonic and hand scalers, polishing cups, polishing brushes, prophy paste [4]. Restorative kits: Spoon excavators, Filling instruments, Cement spatula, Restorative materials [GIC, temporary restorative materials, cavity liners] matrix bands etc. [5]. Surgical kit- Periosteal Elevators, Extraction forceps. Administrative equipment: laptop, informed consent form, feedback form waste transportation boxes will also be carried for the home visit [8].

The dental treatment delivered by DOHCS will include oral prophylaxis, preventive treatments, restoration of carious teeth, treatment for tooth sensitivity, simple extraction of teeth, smoothening of sharp or rough edges of teeth and drug prescriptions for dental pain, abscess or ulcerations. Prosthodontic, endodontic treatments and implants are currently out of scope of the planned DOHCS. Flowchart illustrating care pathway for DOHCS is illustrated in Figure 2.

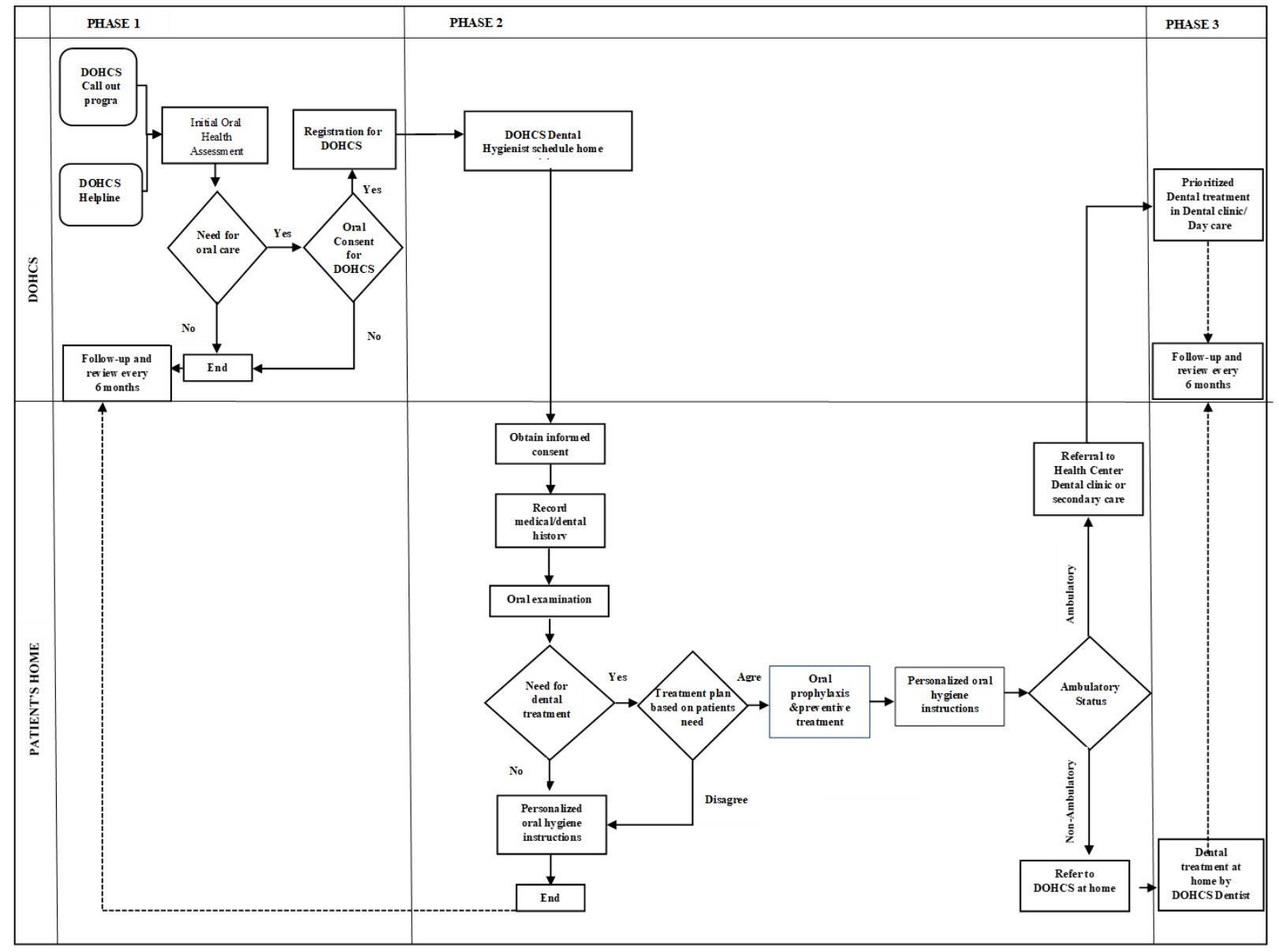

Figure 2 Flowchart illustrating the care pathway for DOHCS 


\section{DOHCS Flow up}

DOHCS team will schedule home visits, once in every 6 months after completed of preventive and/or dental treatment for periodic review, routine examination and reinforcement of oral health education for the elderly and their caregivers.

\section{Benefits of DOHCS}

Delivering DOHCS will ensure targeted prevention and treatment of oral diseases and follow up, for the maintenance of oral health for the elderly in Qatar. Access to regular oral health care will further reduce the likelihood and severity of developing oral diseases in future. It will help the older adults to preserve more natural teeth and functional dentition. Patients with dentures [partial or complete] will experience less episodes of denture stomatitis due to increased awareness, knowledge and reinforcements on maintenance of dentures. It will also help in prevention/ management of oral manifestation of systemic diseases. Additionally, it will help in establishing positive relationship between DOHCS dental team, other health professionals, elderly and their families/ caregivers and encourage them to maintain good health.

This innovative health care delivery system addresses all 6 dimensions of Health care quality improvement described by Institute of Medicine [28]. It is safe, effective, patient-centered, timely, efficient and equitable. DOHCS delivered at home will ensure safety to the elderly and a comfortable experience, as it eliminated the risks associated while accessing oral care facilities, particularly injuries during travel, exhaustion due to long waiting time and stress experienced in the unfamiliar dental clinic setting [29]. These experiences often trigger counterproductive behavior that can make the elderly uncooperative for receiving the necessary care. DOHCS will provide effective and evidence based preventive treatments, following established protocols to help the elderly, their family or care givers to maintain optimum oral health. The treatments rendered will be patient centered and tailored to their individual needs and preferences. It will also eliminate the time constraints of keeping up with appointment slots, which is typically encountered in dental clinics. Hence, empathetic care focusing on their needs and feelings by practicing active listening establishes a socio-emotional connection between the DOHCS dental team and the elderly which is essential for developing and sustaining therapeutic relationship [30]. The attention, warmth, care, concern, and practical assistance, as well as accurate and open communication, can make a tremendous difference to the health of older patients [31].

Availability of DOHCS upon request, throughout the year and prioritized treatment facilities, will reduce the potentially harmful consequences of delays in seeking dental appointments at traditional clinic. Efficient utilization of Mobile Dental Units will ensure that elderly have access to oral care at home, which they would have otherwise neglected or delayed. DOHCS are committed to equity in providing care, as it will be available to all elderly irrespective of their ethnicity, gender or socio-economic status.

The growing phenomenon of ageing was first experienced in countries in Europe, North America, Oceania and some Asian countries, but is now a worldwide concern [32]. This has resulted in inclusion of Geriatric Dentistry in the undergraduate dental curriculum to allow the future Dentists, Dental Hygienists, and Dental Therapists to acquire knowledge about ageing and the necessary skills to develop a better attitude towards this population group. Qatar today is also facing the current demographic trend. The dental professionals must develop the competency to respond to the needs of the elderly while being trained in interprofessional collaboration. This could be achieved by incorporating Geriatric Dentistry in undergraduate curriculum and Continued Professional Development Programs to train the dental workforce in Qatar to increasingly partake in DOHCS and address the oral care needs of the elderly.

\section{Conclusion}

This article describes the proposal for a model for DOHCS with emphasis on collaboration and coordination of care. The proposed program has abundant potential to make a significant impact on improving the oral health of the elderly people in Qatar. Once it is successfully implemented, the program can be scaled up to benefit patients with special needs residing in old age homes, nursing homes and residential care facilities. Owing to absence of such oral health care services till date, the vision of DOHCS is to provide safe, compassionate and essential oral care, through a community based oral health promotion program, that would contribute to achieving the objective of "Healthy ageing" for the people of Qatar enabling them to maintain oral health and lead a comfortable life in old age. The model of DOHCS proposed, employing telephone call out program and hotline, has the potential to be adopted by other specialties like nursing and physiotherapy to ensure universal need responsive preventive and therapeutic services in the respective fields for achieving holistic health for the elderly in Qatar. 


\section{Compliance with ethical standards}

\section{Disclosure of conflict of interest}

Authors declare no conflicts of interest associated with this manuscript.

\section{References}

[1] National Health Strategy 2018 - 2022. Ministry of Public Health. https://www.moph.gov.qa/english/strategies/National-Health-Strategy-2018-2022. Assessed on 21/10/2020.

[2] United Nations. World population Ageing Highlights. Department of Economic and Social affairs. ST/ESA/SER.A/430. 2019.

https://www.un.org/en/development/desa/population/publications/pdf/ageing/WorldPopulationAgeing 2019. Assessed on 21/10/2020.

[3] Qatar demographics. https://www.worldometers.info/population. Assessed on 21/10/2020.

[4] Glassman P, Harrington M, Namakian M, Subar P. Interprofessional Collaboration in Improving Oral Health for Special Populations. Dental Clinics of North America, .2016; 60 [4]: 843-855.

[5] Qatar Old-age dependency ratio. 2020. https://www.statista.com/statistics/961141/qatar-old-agedependency-ratio. Assessed on 21/10/2020.

[6] HMC News. November 2019. https://www.hamad.qa/EN/news/2019/November/Pages/HMCsMultidisciplinary-Home-Care-Teams-Caring-for-More-than-1850-Elderly-Patients-Each-Month.aspx

[7] Fiske J, Lewis D. Unlocking barriers to care. The Development of Standards for Domiciliary Dental Care Services: Guidelines and Recommendations. Report of BSDH Working Group. Gerodontology. 2000; 17[2]: 119-122.

[8] Guidelines for the delivery of a Domiciliary Oral Health Care service. British Society for Disability and Oral Health. 2009.

[9] All Wales Special Interest Group. Special Oral Health Care Guidelines for the delivery of a Domiciliary Oral Health Care service. J Disabil Oral Health. 2006; 7: 166-172.

[10] Lewis A, Kitson A, Harvey G. Improving oral health for older people in the home care setting: An exploratory implementation study. Australasian Journal on Ageing. 2016: 35 [4]: 273-280.

[11] Petersen PE, Yamamoto T. Improving the oral health of older people: the approach of the WHO Global Oral Health Programme. Community Dent Oral Epidemiol. 2005; 33: 81-92.

[12] Ageing and Dental Health- American Dental Association. https://www.ada.org/en/member-center/oral-healthtopics/aging-and-dental-health. Assessed on 20/10/2002.

[13] Lockhart PB et. al. Poor oral hygiene as a risk factor for infective endocarditis-related bacteremia. J Am Dent Assoc. 2009; 140[10]: 1238-1244.

[14] Kanzigg LA, Hunt L. Oral Health and Hospital-Acquired Pneumonia in Elderly Patients: A Review of the Literature. J Dent Hyg 2016; 90[1]: 15-21.

[15] Miranda AF, Freitas Lima SMD, Berto Rezende TM. Fragile elderly, systemic conditions and dental homecare - a mini-review. Arch Gerontol Geriatr Res. 2020; 5[1]: 17-21.

[16] Esterson J, Bazile Y, Mezey M, Cortes, TA, Huba GJ. Ensuring specialty nurse competence to care for older adults, Journal of Nursing Administration. 2013; 43[10]: 517-523.

[17] Simon L. Overcoming historical separation between oral and general health care: Interprofessional collaboration for promoting health equity. AMA J. Ethics. 2016; 18 [9]: 941-949.

[18] Wang TF, Huang CM, Chou C, Yu S. Effect of oral health education programs for caregivers on oral hygiene of the elderly: a systemic review and meta-analysis. Int J Nurs Stud. 2015; 52: 1090-1096.

[19] Coker E, Ploeg J, Kaasalainen S. The effect of programs to improve oral hygiene outcomes for older residents in long-term care: a systematic review. Res Gerontol Nurs. 2014; 7: 87-100. 
[20] Kuo YW, Yen M, Fetzer S, Lee JD. Toothbrushing versus toothbrushing plus tongue cleaning in reducing halitosis and tongue coating: a systematic review and meta-analysis. Nurs Res. 2013; 62: 422-429.

[21] McGrath C, Zhang W, Lo EC. A review of the effectiveness of oral health promotion activities among elderly people. Gerodontology. 2009; 26: 85-96.

[22] NHCPRC, Healthy China Action Plan [2019-2030] by the National Health Commission of the People's Republic of China. 2019.

[23] Kossioni AE et.al. An Expert Opinion from the European College of Gerodontology and the European Geriatric Medicine Society: European Policy Recommendations on Oral Health in Older Adults. J Am Geriatr Soc 2018; 66: 609-613.

[24] Sweeney MP, Manton S, Kennedy C, Macpherson LMD, Turner S. Provision of domiciliary dental care by Scottish dentists: a national survey. British Dental Journal. 2007; 202.

[25] Hummel J, Phillips KE, Holt B, Hayes. Oral health: an essential component of primary care. White paper. June 2015. http://www.niioh.org/sites/default/files/Oral_Health_white_paper

[26] Lewis A, Kitson A, Harvey G. Improving oral health for older people in the home care setting: An exploratory implementation study. Australasian Journal on Ageing. 2016; 35[4].

[27] Tynan A, Deeth L, McKenzie D. An integrated oral health program for rural residential aged care facilities: a mixed methods comparative study. BMC Health Services Research 2018; 18: 515.

[28] Institute of Medicine [IOM]. Crossing the quality chasm: a new health system for the 21st century .Washington, DC : National Academy Press. 2001.

[29] Helgeson M, Glassman P. Oral health delivery systems for older adults and people with disabilities. Spec Care Dentist. 2013; 33[4]: 177-189.

[30] Roter DL, Hall JA. Studies of doctor-patient interaction. Annu Rev Public Health. 1989; 10: 163-80.

[31] Williams SL, Haskard KB, DiMatteo MR. The therapeutic effects of the physician-older patient relationship: Effective communication with vulnerable older patients. Clin Interv Aging. 2007; 2 [3]: 453-467.

[32] Núñez MRR et.al. Teaching undergraduate geriatric dentistry in five South America countries. Gerodontology. 2019; 36: 180-187. 